\title{
SIMULTANEOUS APPROXIMATION PROPERTIES OF DE LA VALLÉE-POUSSIN MEANS IN WEIGHTED ORLICZ SPACES
}

\author{
SADULLA Z. JAFAROV
}

\begin{abstract}
We investigate the simultaneous approximation properties of the de la Vallée-Poussin means in weighted Orlicz spaces in terms of the modulus of smoothness. In terms of the modulus of smoothness the direct theorem of simultaneous approximation is proved. Also, in weighted Orlicz spaces the modulus of smoothness are estimated from below and above in terms of $n$-th partial Fourier sums and de la Vallée-Poussin means.
\end{abstract}

Mathematics subject classification (2010): 30E10, 41A10, 41A25, 46E30.

Keywords and phrases: Boyd indices, weighted Orlicz space, Muckenhoupt weight, modulus of smoothness, de la Vallée-Poussin means.

\section{REFERENCES}

[1] R. AKGÜN, Improved converse theorems and fractional moduli of smoothness in Orlicz spaces, Bull. Malays. Math. Sci. Soc. (2) 36, (2013), no. 1, 49-62.

[2] R. AKGÜN, Approximating polynomials for functions of weighted Smirnov-Orlicz spaces, J. Funct. Spaces Appl. 2012 (2012) Article ID 982360, 41 pp.

[3] R. Akgün, D. M. Israfilov, Polynomial approximation in weighted Smirnov-Orlicz space, Proc. A. Razmadze Math. Inst. 139 (2005), 89-92.

[4] R. AkgÜN, D. M. IsRafiLov, Approximation and moduli of fractional orders in Smirnov-Orlicz classes, Glas. Mat. Ser. III 43 (63) (2008), no. 1, 121-136.

[5] R. AkgÜn, D. M. IsRafilov, Simultaneous and converse approximation theorems in weighted Orlicz spaces, Bull. Belg. Math. Soc., 17 (2010), no. 1, 13-28.

[6] S. P. BAĬBORODov, Approximation of functions by de la Vallée-Poussin sums, Matem. Zametki, 24 (1980), no. 1, 33-47, (in Russian).

[7] C. Bennett And Yu. I. Sharpley, Interpolation of operators, Academic Press, 1988.

[8] A. Böttcher AND YU. I. KARLOVICH, Carleson curves, Muckenhoupt weights and Teoplitz operators, Birkhäuser Verlag, 1997.

[9] D. W. BoyD, Spaces between a pair of reflexive Lebesgue spaces, Proc. Amer. Math. Soc. 18 (1967), 215-219.

[10] D. W. Boyd, Indices of function spaces and their relationship to interpolation, Canad. J. Math. 21 (1969), 1245-1254.

[11] D. W. Boyd, Indices for the Orlicz spaces, Pacific J. Math. 38 (1971), 315-325.

[12] V. T. GAVRILJUK, Linear summability methods for Fourier series and best approximation, Ukrain. Math. Zh. 15 (1963), no. 4, 412-418, (in Russian).

[13] A. Guven, D. M. IsRAfILOV, Approximation by means of Fourier trigonometric series in weighted Orlicz spaces, Adv. Stud. Contemp. Math. (Kyungshang), 19 (2009), no. 2, 283-295.

[14] R. A Devore and G. G. Lorentz, Constructive Approximation, Springer Verlag, 1993.

[15] D. M. Israfilov, Ali Guven, Approximation by trigonometric polynomials in weighted Orlicz spaces, Studia Math. 174 (2006), no. 2, 147-168.

[16] D. M. Israfilov, B. Oktay, R. Akgün, Approximation in Smirnov-Orlicz classes, Glas. Mat. Ser. III 4060 (2005), 87-102.

[17] D. M. Israfilov, R. AkgüN, Approximation in weighted Smirnov-Orlicz classes, J. Math. Kyoto Univ. 46 (2006), no. 4, 755-770. 
[18] D. M. IsRafilov, A. Testici, Simultaneous approximation in Lebesgue spaces with variable exponent, Proc. Inst. Math. Mech. Natl. Acad. Sci. Azerb. 44 (2018), no. 1, 3-18.

[19] D. M. Israfilov, A. Testici, Some inverse and simultaneous approximation theorems in weighted variable exponent Lebesgue spaces, Anal. Math. 44 (2018), no. 4, 475-492.

[20] S. Z. JAFAROv, Approximation by rational functions in Smirnov-Orlicz classes, J. Math. Anal. Appl. 379 (2011), 870-877.

[21] S. Z. Jafarov, Approximation of functions by de la Vallée-Poussin sums in weighted Orlizc spaces, Arab. J. Math. 5 (2016), 125-137.

[22] S. Z. Jafarov, On approximation in weighted Smirnov-Orlicz classes, Complex Var. Elliptic Equ. 57 (2012), no. 5, 567-577.

[23] S. Z. JAFAROv, The inverse theorem of approximation of the function in Smirnov-Orlicz classes, Math. Ineq. Appl. 12 (2012), no. 4, 835-844.

[24] S. Z. JAFAROV, Approximation of conjugate functions by trigonometric polynomials in weighted Orlicz spaces, J. Math. Ineq. 7 (2013), no. 2, 271-281.

[25] S. Z. JAFAROV, Approximation by Fejér sums of Fourier trigonometric series in weighted Orlicz spaces, Hacet. J. Math. Stat. 42 (2013), no. 3, 259-268.

[26] S. Z. JAfarov, On moduli of smoothness of functions in Orlicx spaces, Tbilisi Math. J. 12 (2019), no. 3, 121-129.

[27] M. A. Krasnosel'skiĬ, YA. B. RutickiĬ, Convex Functions and Orlicz Spaces, Translated from the first Russian edition by Leo F. Boron, P. Noordhoff Ltd., Groningen, 1961.

[28] V. M. Kokilashvili, On analytic functions of Smirnov-Orlicz classes, Studia Math. 31 (1968), $43-$ 59.

[29] V. M. KoKILASHVILI, S. G. SAMKO, Operators of harmonic analysis in weighted spaces with nonstandart growth, J. Math. Anal. Appl. 352 (2009), 15-34.

[30] V. M. Kokilashvili, S. G. SAMKo, A refined inverse inequality of approximation in weighted variable exponent Lebesgue spaces, Proc. A. Razmadze Math. Inst. 151 (2009), 132-138.

[31] A. YU. KARLOVICH, Algebras of singular integral operators with piecewise continuous coefficients on reflexive Orlicz spaces, Math. Nachr. 179 (1996), 187-222.

[32] A. YU. KARLOVICH, Singular integral operators with PC coefficients in reflexive rearrangement invariant spaces, Integral Equations Operator Theory 32 (1998), 436-481.

[33] A. YU. KARLOVICH, Fredholmness of singular integral operators with piecewise continuous coeffcients on weighted Banach function spaces, J. Integral Equations Appl. 15 (2003), 263-320.

[34] W. Matuszewska, W. Orlicz, On certain properties of $\varphi$-functions, Bull. Acad. Polon. Sci. Sér. Sci. Math. Astronom. Phys. 8 (1960), no. 7, 439-443.

[35] L. Maligranda, Indices and interpolation, Dissertationes Math. (Rozprawy Mat.) 234 (1985), 49pp.

[36] Le. Yu. Ovsit, A. S. SERdyuk, Approximation of continuous periodic functions by de la Vallée Poussin sums, Zb. Pr. Inst. Mat. NAN Ukr. 8 (1) (2011), 151-161.

[37] J. Prestin, On the approximation by de la Vallée Poussin sums and interpolatory polynomials in Lipschitz norms, Anal. Math. 14 (1987) 251-259.

[38] V. G. Ponomarenko, Approximation of periodic functions in a Orlicz space, Sibirsk. Math. J. 7 (1966), 1337-1346, (in Russian).

[39] A.-R. K. RAmAZANOv, On approximation by polynomials and rational functions in Orlicz spaces, Anal. Math. 10 (1984), 117-132.

[40] M. M. Rao And Z. D. Ren, Theory of Orlicz spaces, Marcel Dekker, New York, 1991.

[41] S. B. StechKin, The approximation of periodic functions by Fejér sums, Tr. Math. Inst. Steklova 62 (1961), 48-60, (in Russian).

[42] S. B. Stechkin, On the approximation of periodic functions by de la Vallée Poussin sums, Anal. Math. 4 (1978), 61-74.

[43] I. I. ShARAPUDINOv, On direct and inverse theorems of approximation theory in vector Lebesgue and Sobolev spaces, Azerb. J. Math. 4 (2014), no. 1, 55-72.

[44] A. S. SeRdyuK, Le. YU. OvsiI, A. P. Musienko, Approximation of classes of analytic functions by de la Vallée Poussin sums in uniform metric, Rend. Mat. Appl. 32 (2012), 1-15.

[45] B. V. Simonov AND S. YU. TiKHONOV, On embeddings of function classes defined by constructive characteristics, Approximation and probability (Bedlewo, Poland 2004), Banach Center Publ. vol. 72, Polish Acad. Sci., Warsaw 2006, pp. 285-307. 
[46] B. V. Simonov, S. Yu. Tikhonov, Embedding theorems in the constructive theory of approximations, Mat. Sb. (2008), 199 (9), 107-148, (in Russian); translation in Sb. Math. 199 (2008), no. 9-10, 1367-1407.

[47] M. F. Timan, Best approximation of a function and linear methods of summing Fourier series, Izv. Akad. Nauk SSSR Ser: Math. 29 (1965), 587-604, (in Russian).

[48] M. F. Timan, Some linear summation processes for Fourier series and best apptroximation, Dokl. Akad. Nauk SSSR 145 (1962), 741-743.

[49] CH. LA. ValléE Poussin, Sur la meilleure approximation des fonctions d'une variable reelle par des expressions d'ordre donné, C. R. Acad. Sci., Paris 166 (1918), no. 4, 799-802.

[50] Y. E. YILDIRIR, D. M. IsRAFILOV, Simultaneous and converse approximation theorems in weighted Lebesgue spaces, Math. Inequal. Appl. 14 (2011), no. 2, 359-371.

[51] A. A. ZAKHAROV, Bound on deviations of continuous periodic functions from their de la ValléePoussin sums, Matem. Zametki, 3 (1968), no. 1, 77-84, (in Russian). 